\title{
Channel Assignment Optimisation Using a Hyper-heuristic
}

\author{
Graham Kendall and Mazlan Mohamad \\ Automated Scheduling, Optimisation and Planning (ASAP) Research Group, \\ University of Nottingham, School of Computer Science and IT \\ Nottingham NG8 1BB, UK \\ E-mail:gxk|mxm@,cs.nott.ac.uk
}

\begin{abstract}
The channel assignment problem is a real world problem from the mobile communications industry. The primary objective is to find the minimum frequency bandwidth given different traffic demand distribution within the mobile network. Besides fulfilling the channel demand requirement, we must also consider the minimum channel reuse distance in order to avoid the effect of call interference within the same cell or adjacent cells. Previous approaches have used graph-colouring algorithms, heuristic search, neural networks, simulated annealing and genetic algorithms (GA). In this paper, we propose a hyperheuristic methodology and compare our approach against channel assignment problems from the literature.
\end{abstract}

Index Terms - Channel Assignment, Mobile Communication, Adaptive Heuristic Search, Hyper-heuristic, Optimisation.

\section{INTRODUCTION}

The technology supporting mobile communications has grown tremendously and continues to do so. The key aspect of this growth is due to the popularity of mobile communications, which is the ability to provide instant connectivity anytime and to anywhere in the world. Starting from an analog standard (known as $1^{\text {st }}$ generation), we can see the full migration from an analog into a digital standard (known as $2^{\text {nd }}$ generation) throughout the world. The $2^{\text {nd }}$ generation mobile standard (GSM, D-APMS and CDMA), with high quality voice and the capability to provide highspeed data services to the mobile user as an additional service, are the driving factors behind the high growth of sales in communication technology. The mobile communication has recently introduced a $3^{\text {rd }}$ generation mobile wireless technology with the aim to have a global standard for all applications and countries.

The Federal Communications Commission (FCC), in their respective countries, controls the operating of mobile telephone networks and they issue licenses to mobile operators, which means the mobile operators, are allowed to use only the frequency bandwidth allocated to them. The allocated frequency bandwidth is divided into sets of carriers or channels using different techniques such as frequency division (FD), time division (TD), or code division (CD), which each channel uses for call establishment.

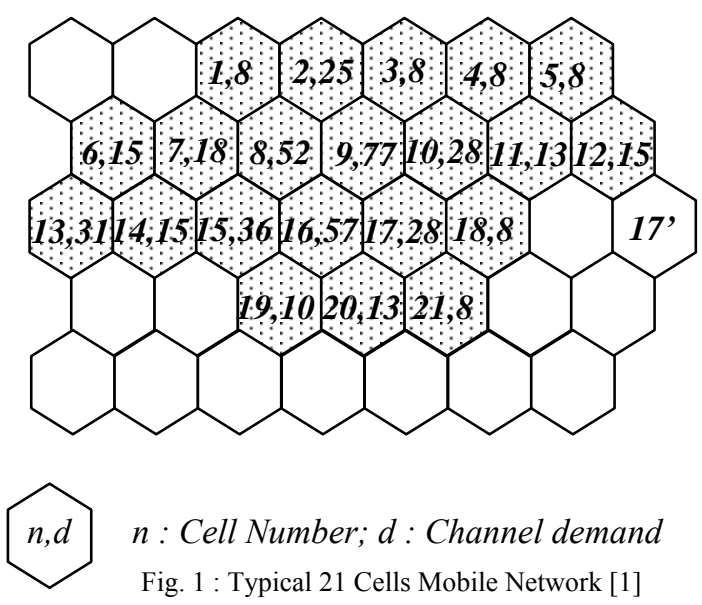

Mobile users in a particular cell use the channel that is allocated at the base station. A channel can simultaneously be used at another base station, subject to a minimum reuse distance, i.e., there is enough distance between the base stations in order to provide interference free communication.

In mobile communication, all the information from mobile sets (MS) to the base stations (BS) travels via radio channels. The geographical area covered by a BS is a cell. This can be represented as a hexagonal network (refer to Figure 1 [1]). A mobile communication network consists of thousand of cells in order to cover the required region, each with a BS at the centre. Each BS will have a dedicated radio channel and the number of the channels assigned will be based on the traffic requirement for the particular cell (for example, there will be a higher channel demand in metropolitan areas compared to suburban areas).

Besides the traffic demand requirement, the assignment of radio channels is subject to the three types of interference constraints;

a) Cochannel constraint. The same channel cannot be allocated to certain pairs of radio cells simultaneously. For example consider Fig. 1. Assuming we have mobile set $i$, that uses channel $k$ in cell 17 ; the same channel, $k$ can simultaneously be used in cell $17^{\prime}$ by another mobile set $j$, if mobile set, $i$ and mobile set, $j$ are separated by minimum reuse distance (in this case 3 ). 
b) Adjacent channel constraint. The same adjacent channel cannot be allocated to adjacent radio cells simultaneously. Assuming we have mobile set $i$, that uses channel $k$ in cell 17 , if, we have mobile set, $l$, that uses channel $g$ in an adjacent cell (say cell 9), we can comply with adjacent channel constraint if channel $k$ and channel $g$ are separated by a minimum channel separation distance.

c) Cosite constraint. Any pair of channels assigned in the same cell must be separated by a minimum channel separation distance.

The channel demand distribution over the whole region of a mobile network varies from cell to cell, according to a nonuniform distribution of business and social activities [1]. In. Fig.1, we can see the highest channel demand is 77 channels at cell 9 and the lowest channel demand is 8 for several cells $(1,3,4$, etc $)$. Mobile operators have to fully utilise a given limited spectrum bandwidth, which can satisfy mobile customers by avoiding or minimising call blocking especially in high channel demand cells (cell 9,16,etc.). With a limited frequency spectrum allocated to the system, the efficiency in which of radio channels are assigned is very important to overcome congestion problems in the network.

The assignment of radio channel is generally called the Channel Assignment Problem (CAP). The CAP can be viewed as a combinatorial optimisation problem, with the difficulty of CAP increasing exponentially with the problem size. There are three kinds of channel assignment; Fixed Channel Assignment (FCA), Dynamic Channel Assignment (DCA) and Hybrid Channel Assignment (HCA) [2]. In FCA, the radio channels are assigned permanently to each cell based on a predefined channel demand. In DCA the channels are dynamically assigned to cells based on channel requests. DCA provides flexibility and traffic adaptability at the cost of higher complexity, but under heavy traffic conditions, DCA strategies are less efficient compared to FCA [3]. Since heavy traffic condition is expected in the future, the efficiency of an FCA scheme is highly desirable [2]. HCA is a combination between FCA and DCA. In this paper, we will consider only FCA schemes.

Mobile customers can make a call using a channel of the cell in which the user is currently situated. Under heavy traffic conditions, the network planning engineers have to reduce the cell size (cell splitting) and use advanced antenna technology in order to cater for the increase in channel demand. This causes the number of base stations to increase. Under FCA, the CAP objective is to optimise the assignment of the limited channel resources to a base station without ignoring service quality such as cochannel and adjacent interference. Using an exhaustive search strategy, the optimum result could be obtained, but as the number of base station increases, finding an optimum solution is extremely difficult [4].

In this paper, we will introduce a hyper-heuristic approach to tackle this problem and present computational results.

\section{RELATED WORK}

The channel assignment problem continues to be an active research area [5]. Previous approaches to solve CAP can be categorised as approaches using graph theory [6], heuristics $[1,7,8,9]$, local search [10], neural networks $[11,12,13]$, the utilisation of simulated annealing [14] and genetic algorithms [15].

Graph theory approaches used the sequential assignment of channels based on assignment difficulty. Zoellner and Bell [6] used a frequency-exhaustive strategy or a requirementexhaustive strategy to assign a channel to cells which were ranked in node-degree order or node-colouring order.

Using an heuristic approach, Box [7], used the principle of assignment difficulty to arrange the cell sequence or ordering. With this sequence, he used a simple iterative method to assign the channel. Sivarajan et. al [9], produced eight CAP algorithms by ranking the calls in row-wise ordering or column-wise ordering and the cells in node-degree order or node-colouring order. With each rank, they used a requirement exhaustive strategy and a frequency exhaustive strategy to assign the channel. Chakraborty [1] proposed an algorithm for generating a pool of valid solutions. He claimed that the algorithm is very fast and could find optimum or near optimum solutions.

Wang and Rushforth [10] used a local search approach. In the feasible region $\mathrm{R}$ with the current solution $X_{c}$, the next solution , $X_{c+1}$ will be accepted if the cost function $f\left(X_{c+1}\right)<$ $f\left(X_{c}\right)$. Based on benchmark test results, this approach produced superior results to those reported in the literature.

A neural network approach was proposed by Kunz [11] and the result was improved by Funabiki and Takefuji [12]. Subsequently, Kim et. al [13], proposed a modified discrete Hopfield neural network in order to escape from local optima. They formulated CAP as an energy-minimisation problem and claimed that the performance is better when compared to previous approaches. The simulated annealing approach [14], guarantees to find the global optimum but with the disadvantage that the rate of convergence is slow.

A genetic algorithm approach was proposed by Lai and Coghill [15] who investigated the channel assignment problem using a computational technique that mimics the evolutionary process. The primary goal in solving CAP is to satisfy cosite and cochannel constraints and satisfy all channel demands required by each cell. They used a string structure to represent the channel required for each base station, where the total length of each string is the sum of channels required. The evolutionary process uses partially matched (PMX) crossover and basic mutation, with two extra parameters in order to bias cosite and cochannel constraints in their fitness function. They claim that their approach is elegant and simple.

\section{PROBLEM DEFINITION}

Frequency bandwidths are allocated to cellular service providers with a range of $\left[b_{\min }, b_{\max }\right]$ [16]. The bandwidth is partitioned into a set of channels, $F$ (or often called frequencies) and can be represented as positive integers 1,2 , 
$3, \ldots . f_{\max }$ where $f_{\max }$ is a maximum channel number. A basic channel assignment problem for radio network planning consists of (mostly adopted from [9] and [10])

a. $\quad N$ : The number of cells in the network.

b. $D:$ demand vector, $D=\left(d_{1}, d_{2}, \ldots, d_{N}\right)$ where $d_{i}$ : the number of radio channel required in cell $i$ in order to satisfy channel demand.

c. $C$ : Compatibility matrix, $C=\left(c_{i j}\right)_{N x N}$ denotes the frequency separation required between cell $i$ and cell $j$.

d. $\quad$ Call $_{i k}$ : Cell $i$ with call $k$ where $1 \leq i \leq N, 1 \leq k \leq d_{i}$.

e. $f_{i k}$ : A radio channel is assigned to $\operatorname{Call}_{i k}$, where $f_{i k} C$ $F$.

f. Frequency separation constraint $-\left|f_{i k}-f_{j m}\right| \geq c_{i j}$, for all $i, j, k, m(\mathrm{i} \neq j, k \neq m), c_{i j}$ is defined in Compatibility Matrix, C. If $i=j$, it's become co-site constraint.

Therefore, the channel assignment problem (CAP) is a task to find the minimum bandwidth (span) required by the system i.e.:

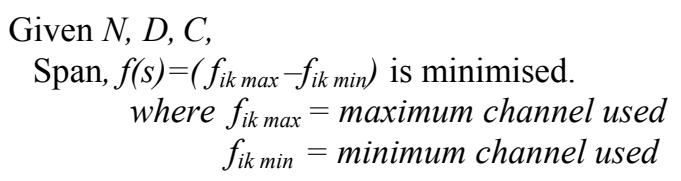

IV. THE ALGORITHM

\section{A. Hyper-heuristic}

We propose using a hyper-heuristic approach to solve the channel assignment problem. A hyper-heuristic is a knowledge poor meta-heuristic that does not use problem specific information [17]. Based on non-domain information, such as CPU time, change in the objective function etc., a hyper-heuristic will decide which low level heuristic to call at each decision point. In order to implement the search, we may use simple neighbourhood heuristics such as delete, add and swap. The advantages of using a hyper-heuristics for practical scheduling and indeed optimisation problems are (mostly adapted from [18]):

- It is easy for the problem owner to consider their problem by modeling it using simple heuristics.

- Simple heuristics are typically faster to implement compared to some other approaches.

- It is robust enough to effectively handle a wide range of problem instances by small modifications or additions.

Hyper-heuristics operate at a higher level of abstraction than meta-heuristics by managing a set of low level heuristics (LLH). The general framework can be shown in figure 2 [17].

Hyper-heuristics are problem independent and have been successfully applied to various optimisation and scheduling problems $[19,20,21,22]$. For example, a genetic algorithm hyper-heuristic [20] used an allele in a chromosome to represent each LLH. The quality of the solution is evaluated based on the sequence of calls within the chromosome. In [21], a Monte Carlo hyper-heuristic approach is used to optimise the scheduling of electrical component placement on a printed circuit board. They used an acceptance criteria to determine solution quality returned by each LLH.

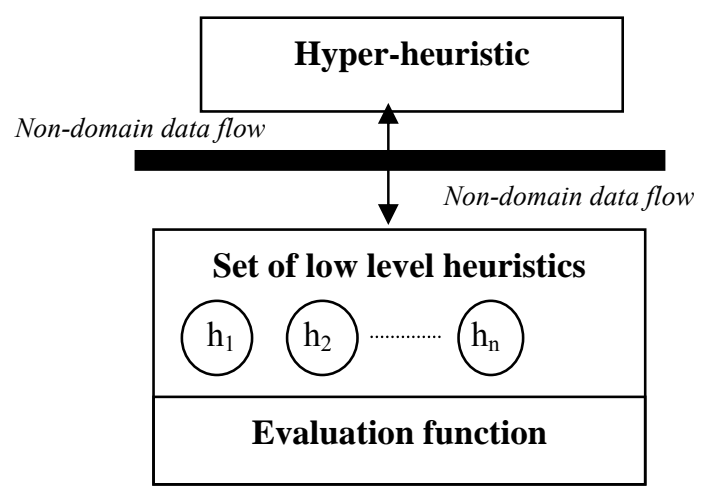

Figure 2: A Hyper-heuristic Framework

Another approach has used a tabu list to monitor the performance of each LLH in solving an examination timetabling problem [22]. The choice function hyper-heuristic [23] ranks the solution quality performance of each LLH. Based on the quality of the previous result; the hyper-heuristic will decide which LLH should be called next.

Let us use the local search framework to define:

Solution Space, $S$ : The set of possible ordered list of calls.

Objective function: $f\left(S_{\max }\right) \in F$, the maximum frequency for solution $S_{\max } \in \mathrm{S}$.

$N$ : neighbourhood structure.

$H$ : A set of Low Level Heuristic $\left(\mathrm{h}_{1}, \mathrm{~h}_{2}, . . \mathrm{h}_{\mathrm{n}}\right)$

The hyper-heuristic algorithms is as follows:

Step 1: (Initialisation)

(A) Choose Initial solution $S_{0} E S$;

(B) Record the best obtained solution, $S_{\text {best }}=S_{0}$ and $f\left(S_{\text {best }}\right)=f\left(S_{0}\right)$;

Step 2: (Choice and termination)

(A) Randomly choose an $h_{c} \in H$;

(B) Apply $h_{c}$ to produce $S_{c} \in N\left(S_{0}\right)$;

(C) Compute $\delta=f\left(S_{c}\right)-f\left(S_{0}\right)$;

(D) If the acceptance criteria is true, then accept $S_{c}$ (and proceed to Step 3);

(E) If $S_{c}$ is rejected and stopping condition=false, then return to Step2(A);

(F) Terminate by a stopping condition.

Step 3: (Update)

Re-set $S_{0}=S_{c}$, and if $f\left(S_{c}\right)<f\left(S_{\text {best }}\right)$, perform Step $1(C)$.

Return to Step2 if stopping condition=false.

\section{B. List of Low level heuristic (LLHs)}

Low level heuristics are defined as simple local search operators, which are problem dependent. In this work, we randomly call LLHs to generate a unique move from the current solution to the new solution. The list of LLHs that we use are shown below: 
$\mathrm{h}_{1}$ - Sort the channel from lowest to highest, delete the call with the highest channel assignment, randomly insert at any point and reassign the channel.

$\mathrm{h}_{2}$ - Same as $\mathrm{h}_{1}$, but randomly select the call to delete.

$\mathrm{h}_{3}$ - Same as $\mathrm{h}_{1}$, but find the best point at which to reassign.

$\mathrm{h}_{4}$ - Same as $\mathrm{h}_{1}$, but randomly change the call order starting from insertion point.

Each move will return the current frequency span (which represents the objective function).

All the proposed LLHs will create unique moves with zero violating constraint with faster computation time except for $\mathrm{h}_{3}$, which acts as a steepest decent heuristic. $h_{3}$ will find the best neighbour in the current nighbourhood structure; therefore it requires more computation time.

\section{Acceptance Criteria}

The acceptance of new solution (see 2(D) and 2(E) in above) will be decided by the hyper-heuristic. In this work, we use 4 different types of acceptance criteria. These are:

Accept all moves $(A M)$ - All the returned solutions from the LLHs will be accepted. The acceptance criteria is always true.

Only improving moves (OI) - Only moves which improve on the current solution will be accepted $(\delta<0)$.

Monte Carlo (MC) - The acceptance of worse solution will be based on a probability that decreases with increases of $\delta$ $[21,24]$.

Record-to-Record Travel (RRT) - The idea of the RRT acceptance criteria was proposed by Dueck and is a variant of the Great Deluge acceptance criteria [25]. At each iteration, any configuration is accepted which is not much worse compared to the current solution. In the $R R T$ algorithm [25], Dueck introduced a DEVIATION parameter, which is a small value added to the current solution quality in order to increase the possibility that it is accepted. If $\delta<$ DEVIATION, the algorithm will accept the new solution but will not update the solution quality for future comparison. Based on preliminary tests, the best DEVIATION value used in our experiments is 2 .

\section{EXPERIMENTS AND RESULTS}

\section{A. Benchmarks Instances}

We will use the channel assignment benchmark problems, used in previous works [1], [8]-[12]. They have different levels of problem difficulty. That is they have different engineering interference, different traffic demands and different network sizes. We will consider three different network sizes, 21, 25 and 55, which are the common sizes used by other researchers [1].

As reported in [1], some of the benchmarks (which can be considered as easy benchmark problems), can be solved to optimality. Taking this into consideration, we only consider those benchmarks (difficult problems) where optimal results are still unknown.

With different cluster sizes, we can set up different test environments using different $C_{i i}$ values, and different values for $\alpha$ and $\beta$ which corresponds to minimum reuse distance and degree of interference between cells. The compatibility matrices we use are shown in table I [1]. These are used in conjunction with the traffic demands shown in table II. The traffic demand has uniform and non-uniform distribution, and are the values used by many other researchers.

\begin{tabular}{|c|c|c|c|c|c|c|c|}
\hline No & $\begin{array}{l}\text { Cluster } \\
\text { Size (N) }\end{array}$ & $\alpha$ & $\beta$ & $\begin{array}{l}\text { Distance } \\
\text { cell } i \text { and } \\
\text { cell } j\end{array}$ & $\begin{array}{l}C_{i j} \\
\text { entry }\end{array}$ & $C_{i i}$ & $C_{i j}$ name \\
\hline 1 & 21 & 2 & 2 & $\begin{array}{l}1 \\
2 \\
>2\end{array}$ & $\begin{array}{l}2 \\
1 \\
0\end{array}$ & 6 & C4_21 \\
\hline 2 & 21 & 2 & 2 & $\begin{array}{l}1 \\
2 \\
>2\end{array}$ & $\begin{array}{l}2 \\
1 \\
0\end{array}$ & 5 & C6_21 \\
\hline 3 & 21 & 2 & 1 & $\begin{array}{l}1 \\
2 \\
>2 \\
\end{array}$ & $\begin{array}{l}1 \\
1 \\
0 \\
\end{array}$ & 4 & C7_21 \\
\hline 4 & 21 & 2 & 2 & $\begin{array}{l}1 \\
2 \\
>2 \\
\end{array}$ & $\begin{array}{l}2 \\
1 \\
0 \\
\end{array}$ & 4 & C8_21 \\
\hline 5 & 25 & 2 & 1 & $\begin{array}{l}1 \\
2 \\
>2 \\
\end{array}$ & $\begin{array}{l}1 \\
1 \\
0\end{array}$ & 2 & C1_25 \\
\hline 6 & 55 & 2 & 1 & $\begin{array}{l}1 \\
2 \\
>2\end{array}$ & $\begin{array}{l}1 \\
1 \\
0\end{array}$ & 7 & C1_55 \\
\hline
\end{tabular}

TABLE I : COMPATIBILITY MATRIX

Note: We use the same notation for $C_{i j}$ name $\left(6^{\text {th }}\right.$ column $)$ as in [1].

\begin{tabular}{|l|l|}
\hline Cell Size & Traffic Demand \\
\hline 21 & $D 1 \_21:\{825888151852772813153115365728810$ \\
& $138\}$ \\
& $D 2 \_21:\{55581225302530404045203025151530$ \\
& $202025\}$ \\
\hline 25 & $D 3 \_25:\{1011959457488910776455764575\}$ \\
& $D 4 \_25:\{55581225302530404045203025151530$ \\
& $2020258555\}$ \\
\hline 55 & $D 5 \_55:\{55581225302530404045203025151530$ \\
& 20202585555558122530253040404520302015 \\
& $15302020258555258555\}$ \\
& $D 6 \_55:\{101195945748891077645576457510$ \\
& $1195945748891077645576457564575\}$ \\
\hline
\end{tabular}

Note: The values in the $2^{\text {nd }}$ column represent the number of channels needed in each cell.

\section{B. Results}

We have implemented and tested our algorithm on a Pentium III-700 MHz computer. We compare our performance with [1], which proposed an algorithm that generates a population of random valid solutions using a 
quadnary representation $[0,+1,-1,+9]$, which means [assignable,used,unassignable,unused].

Another comparison is with recent work by Ghosh et al. [26], in which they use a genetic algorithm based on geometric symmetry. We run our experiments for 400 seconds for each benchmark problem.
Using a random constructive heuristic to generate an initial solution and using 'runtime expired' as our stopping criteria, we ran each experiment 10 times. By applying our approach we were able to achieve promising results as presented in table III ( $A M$ and $O I)$ and table IV (MC and $R R T)$.

\begin{tabular}{llllllll}
\hline Test & $\begin{array}{l}\text { C_Matric(C)/ } \\
\text { Demand(D) }\end{array}$ & $\begin{array}{l}\text { Trivial } \\
\text { Lower } \\
\text { Bound }\end{array}$ & $\begin{array}{l}\text { Intial } \\
\text { solution }\end{array}$ & $\begin{array}{l}\text { Chakraborty } \\
{[1]}\end{array}$ & $\begin{array}{l}\text { Ghosh et al. } \\
{[26]}\end{array}$ & $\begin{array}{l}\text { AM Hyper-heuristic } \\
\text { (span/ } \% \\
\text { improvement) }\end{array}$ & $\begin{array}{l}\text { OI Hyper-heuristic } \\
\text { (span/ \% } \\
\text { improvement })\end{array}$ \\
\hline 1 & C4_21/D1_21 & 457 & 622 & 457 & - & $457 / 26.5$ & $457 / 26.5$ \\
2 & C4_21/D2_21 & 265 & 337 & 280 & & $272 / 19.2$ & $270 / 19.8$ \\
3 & C6_21/D1_21 & 381 & 595 & 463 & 427 & $448 / 24.7$ & $446 / 25.4$ \\
4 & C6_21/D2_21 & 221 & 316 & 273 & 253 & $268 / 15.2$ & $266 / 15.8$ \\
5 & C7_21/D1_21 & 305 & 353 & 305 & - & $305 / 13.6$ & $305 / 13.6$ \\
6 & C7_21/D2_21 & 177 & 226 & 197 & - & $185 / 18.1$ & $184 / 18.5$ \\
7 & C8_21/D1_21 & 305 & 507 & 465 & - & $447 / 11.8$ & $442 / 12.8$ \\
8 & C8_21/D2_21 & 177 & 320 & 278 & - & $275 / 14.1$ & $73 / 15.0$ \\
9 & C1_25/D3_25 & 21 & 78 & 73 & - & $73 / 6.4$ & $200 / 4.3$ \\
10 & C1_25/D4_25 & 89 & 209 & 121 & - & $200 / 4.3$ & $309 / 8.0$ \\
11 & C1_55/D5_55 & 309 & 336 & 309 & - & $309 / 8.0$ & $72 / 26.5$ \\
12 & C1_55/D6_55 & 71 & 98 & 79 & - & $73 / 25.5$ & \\
\hline
\end{tabular}

TABLE III : AN AVERAGE RESULT OF TEN RUNS FOR ALL MOVES AND ONLY IMPROVING

\begin{tabular}{llllllll}
\hline Test & $\begin{array}{l}\text { C_Matric(C)/ } \\
\text { Demand(D) }\end{array}$ & $\begin{array}{l}\text { Trivial } \\
\text { Lower } \\
\text { Bound }\end{array}$ & $\begin{array}{l}\text { Intial } \\
\text { solution }\end{array}$ & $\begin{array}{l}\text { Chakraborty } \\
{[1]}\end{array}$ & $\begin{array}{l}\text { Ghosh et al. } \\
{[26]}\end{array}$ & $\begin{array}{l}M C \text { Hyper-heuristic } \\
\text { (span/\% } \\
\text { improvement) }\end{array}$ & $\begin{array}{l}R R T \text { Hyper-heuristic } \\
\text { (span/ \% } \\
\text { improvement) }\end{array}$ \\
\hline 1 & C4_21/D1_21 & 457 & 622 & 457 & - & $457 / 26.5$ & $\mathbf{4 5 7 / 2 6 . 5}$ \\
2 & C4_21/D2_21 & 265 & 337 & 280 & & $270 / 19.8$ & $\mathbf{2 7 0 / 1 9 . 8}$ \\
3 & C6_21/D1_21 & 381 & 595 & 463 & 427 & $443 / 25.5$ & $\mathbf{4 3 7 / 2 6 . 0}$ \\
4 & C6_21/D2_21 & 221 & 316 & 273 & 253 & $266 / 15.8$ & $\mathbf{2 6 1 / 1 7 . 4}$ \\
5 & C7_21/D1_21 & 305 & 353 & 305 & - & $305 / 13.6$ & $\mathbf{3 0 5 / 1 3 . 6}$ \\
6 & C7_21/D2_21 & 177 & 226 & 197 & - & $184 / 18.6$ & $\mathbf{1 8 2 / 1 9 . 5}$ \\
7 & C8_21/D1_21 & 305 & 507 & 465 & - & $444 / 12.4$ & $\mathbf{4 3 5 / 1 4 . 2}$ \\
8 & C8_21/D2_21 & 177 & 320 & 278 & - & $274 / 14.4$ & $\mathbf{2 6 8 / 1 6 . 2}$ \\
9 & C1_25/D3_25 & 21 & 78 & 73 & - & $73 / 6.4$ & $\mathbf{7 3 / 6 . 4}$ \\
10 & C1_25/D4_25 & 89 & 209 & 121 & - & $200 / 4.3$ & $\mathbf{2 0 0 / 4 . 3}$ \\
11 & C1_55/D5_55 & 309 & 336 & 309 & - & $309 / 8.0$ & $\mathbf{3 0 9 / 8 . 0}$ \\
12 & C1_55/D6_55 & 71 & 98 & 79 & - & $72 / 26.5$ & $\mathbf{7 1 / 2 7 . 6}$ \\
\hline
\end{tabular}

TABLE IV : AN AVERAGE RESULT OF TEN RUNS FOR MONTE CARLO AND RECORD-TO-RECORD TRAVEL

\section{DISCUSSION}

When we compare the results in Table III and table IV, we can see that the performance of the $R R T$ hyper-heuristic is superior compared to the other approaches. This suggests that by controlling the margin of the acceptance solution quality, it will lead to better results. In $R R T$, if the solution quality is worse, compared to the incumbent solution, but within the 'margin limit', it will become a new configuration for the next iteration.

When we compare test 3 and test 4 which are the most difficult benchmark problems, even though our proposed algorithms cannot beat the existing result from [26], their solution took between $16-80$ hours to produce such good quality solutions. Our result is promising especially for RRT, which is achieving an improvement of $26 \%$ and $17 \%$ when compared to the initial solution.

For other test problems, when we compare our result with constructive heuristics as proposed in [1], all our proposed hyper-heuristic manage to find a better solution except for test 10.

\section{CONCLUSIONS AND FUTURE WORK}

The channel assignment problem is a real world problem from the mobile communications industry. The primary objective is to find the minimum frequency bandwidth given different traffic demand distribution within the mobile network. Besides fulfilling the channel demand requirement, we must also consider the minimum channel 
reuse distance in order to avoid the effect of call interference within the same cell or adjacent cells.

In this work, we have proposed a hyper-heuristic methodology which is problem independent. Unlike other meta-heuristic approaches such as variable neighbourhood search; we can freely move from one neighbourhood to another neighbourhood structure. Therefore, we see the motivation of using a hyper-heuristics as a rapid development tool for optimisation problems.

We have proposed a hyper-heuristic with four different acceptance criteria. Based on experimental result, the $R R T$ hyper-heuristics is superior compared to the other proposed hyper-heuristics. Our future interest is to use different strategies to produce initial solutions, using intelligent LLHs (LLHs which themselves have an acceptance criteria, based on random descent and steepest descent local search).

\section{REFERENCES}

[1] G. Chakraborty, An Efficient Heuristic Algorithm for Channel Assignment Problem in Cellular Radio Networks, IEEE Trans on Veh Technology, vol. 50, no. 6, pp. 1528-1539, Nov 2001.

[2] D. Corne, M.J. Oates and G.D Smith, Telecommunication Optimisation : Heuristic and Adaptive Techniques, John Wiley \& Sons, 2000.

[3] I. Katzela and M. Naghshineh, Channel Assignment Schemes for Cellular Mobile Telecommunication Systems - A Comprehensive Survey, IEEE Personal Communications Magazine, June 1996.

[4] T. Fujii and N. Nakazima, Radio Channel Assignment System for Cellular Mobile Communications, Electronics and Communications in Japan, Part 1, Vol. 86, No. 5, 2003.

[5] X.N. Fernando and A.O. Fapojuwo, A Viterbi-Like Algorithm with Adaptive Clustering for Channel Assignment in Cellular Radio Networks, IEEE Trans on Veh Technology, vol 51, pp. 73-87, January 2002.

[6] J.A Zoellner and C.A Beall, A breakthrough in spectrum conserving frequency assignment technology, IEEE Trans. Electromagnetic Comp.,vol. EMC-19, pp. 313-319, Aug. 1977.

[7] F. Box, A Heuristic technique for assigning frequencies to mobile radio networks, IEEE Trans on Veh Technology, vol. VT-27, pp. 57-64, 1978.

[8] A. Gamst and W. Rave, On the frequency assignment in mobile automatic telephone system, in Proc. IEEE GLOBECOM'82, 1982, pp. 309-315.

[9] K.N. Sivarajan, R.J. McEliece and J.W.Ketchum, Channel assignment in cellular radio, in Proc. $39^{\text {th }}$ IEEE Veh. Technology. Soc. Conf., pp. 846-850, May 1989.

[10] W. Wang and C.K Rushforth, An Adaptive Local-Search Algorithm for the Channel-Assignment Problem (CAP), IEEE Trans on Veh Technology, vol 45, pp. 459-446, August 1996.

[11] D. Kunz , Channel Assignment for Cellular Radio Using Neural Networks, IEEE Trans on Veh Technology, vol 40, pp. 118-193, Feb 1991.

[12] N. Funabiki and Y. Takefuji, A Neural Network Parallel Algorithm for Channel Assignment Problems in Cellular Radio Network, IEEE Trans on Veh Technology, vol. 41, no. 4, pp. 430-437, November 1986.

[13] J.S. Kim, S.H. Park, P.W. Dowd and N.M. Nasrabadi, Cellular radio channel assignment problems in cellular networks, IEEE Trans on Veh Technology, vol 46, pp. 957-967, Nov. 1996.

[14] M. Duque-Anton, D. Kunz and B. Ruber, Channel Assignment for Cellular Radio Using Simulated Annealing, IEEE Trans on Veh Technology, vol 42, pp. 14-21, Feb 1993.

[15] W.K. Lai, G.G. Coghill, Channel Assignment Through Evolutionary Optimisation, IEEE Trans on Veh Technology, vol 45, No.1, pp. 91-95, Feb 1996.

[16] A. Eisenblatter and A. Koster, FAP website [online]. Available: http://fap.zip.de/
[17 ] E. Burke, E. Hart, G. Kendall, J. Newall, P. Ross, S.Schulenburg, Hyper-heuristics: An Emerging Direction In Modern Search Technology, Ch. 16, Handbook of Meta-Heuristics (eds. F.Glover and G.Kochenberger), pp 457-474, Kluwer 2003.

[18] P. Cowling, G. Kendall and E. Soubeiga, A Hyperheuristic Approach to Scheduling a Sales Summit, In LNCS 2079, Practice and Theory of Automated Timetabling III : Third International Conference, PATAT 2000, Konstanz, Germany, August 2000, selected papers (eds Burke E.K. and Erben W), Springer-Verlag, pp 176-190, ISBN : 3-540-424210 .

[19] E.K Burke, G Kendall and E Soubeiga, A Tabu-Search Hyper-Heuristic for Timetabling and Rostering. Journal of Heuristics, 9(6), pp. 451470, 2003.

[20] L. Han and G. Kendall, Investigation of a Tabu Assisted HyperHeuristic Genetic Algorithm. In proceedings of Congress on Evolutionary Computation(CEC2003), vol. 3, pp. 2230-2237, Canberra, Australia.

[21] M. Ayob and G. Kendall, A Monte Carlo Hyper-Heuristic To Optimise Component Placement Sequencing For Multi head Placement Machine, InTech'03 Thailand, pp. 132-141, ISBN 974-658-151-1.

[22] G. Kendall and N. Mohd Hussin, An Investigation of a Tabu Search Based Hyper-heuristic for Examination Timetabling, Accepted for publication in selected papers from MISTA2003, Kluwer Publication eds. (G.Kendall, E.Burke and S. Petrovic).

[23] E. Soubeiga, Development and Application of Hyperheuristics to Personnel Scheduling, PhD Thesis, Department of Computer Science, University of Nottingham, UK, June 2003.

[24] F. Glover and M. Laguna, Tabu search, chapter 3. In: Reeves, C. R.(ed) Modern heuristic techniques for combinatorial problems, McGraw-Hill, 1995, pp 70-150.

[25] G. Dueck, New Optimization Hueristics : The Great Deluge Algorithm and the Record-to Record Travel, Journal of Computational Physics, 104, pp. 86-92, 1993.

[26] S.C. Ghosh, B.P. Sinha and N. Das, Channel Assignment Using Genetic Algorithm Based on Geometric Symmetry, IEEE Trans on Veh Technology, vol. 52, no. 4, pp. 860-875, July 2003. 